\title{
On the theory of superconductivity in ferromagnetic superconductors with triplet pairing
}

\author{
V.P. Mineev and T. Champel \\ Commissariat à l'Energie Atomique, DSM/DRFMC/SPSMS \\ 17 rue des Martyrs, 38054 Grenoble Cedex 9, France
}

(November 19, 2018)

\begin{abstract}
We point out that ferromagnetic superconductors with triplet pairing and strong spin-orbit coupling are even in the simplest case at least two-band superconductors. The Gor'kov type formalism for such superconductors is developed and the Ginzburg-Landau equations are derived. The dependence of the critical temperature on the concentration of ordinary point-like impurities is found. Its nonuniversality could serve as a qualitative measure of the two-band character of ferromagnetic superconductors. The problem of the upper critical field determination is also discussed.
\end{abstract}

\section{INTRODUCTION}

The extension of the Bardeen-Cooper-Schrieffer (BCS) theory of superconductivity to the case with two bands of itinerant electrons was developed soon after the appearance of the BCS theory [1]. This theory has been the subject of renewed interest following the recent discovery of $\mathrm{MgB}_{2}$ - the first superconducting material where the existence of two energy gaps has been unambiguously demonstrated by thermodynamic and spectroscopic measurements [2]. Certainly, there are many other superconducting compounds where multiband effects are less pronounced and experimentally invisible because the Cooper pairing occurs mostly in one band of the itinerant electrons or holes. On the other hand, there is a whole class of superconductors where two-band (or more generally multiple band) superconductivity is an inherent property: the so called ferromagnetic superconductors where the different bands with spin "up" and spin "down" electrons are always present. UGe $\mathrm{UG}_{2}[3,4], \mathrm{ZrZn}_{2}$ [5], and URhGe [6] are recent examples of such materials where the superconducting states are expected to be spin triplet in order to avoid the large depairing influence of the exchange field due to ferromagnetism.

The symmetry classification of the superconducting states for itinerant ferromagnetic spin-triplet superconductors has been proposed recently by several authors [7-9]. At the same time, a general Gor'kov-type mathematical description of the multi-band superconductivity in a ferromagnetic metal with a triplet pairing has not been developed yet. The principal goal of this article is to present such a description for a two-band ferromagnetic metal with an anisotropic spectrum of quasiparticles and a general form of the pairing interaction. Being useful for a concrete calculation with a particular form of the spectrum and the pairing interaction, this approach allows to solve in general terms several problems typical for the superconductivity theory, such as: the critical temperature determination, the derivation of the Ginzburg-Landau equations, the suppression of the superconductivity by impurities, the upper critical field calculation.

We begin with the general form of the order parameter and the pairing interaction in a two-band itinerant ferromagnet. Then the Gor'kov equations will be written. That permits to calculate the spectrum of the quasiparticles and the critical temperature, and to derive the system of the coupled equations for the order parameters from two bands. Then, a law of suppression of the critical temperature by point-like nonmagnetic impurities is found. Its characteristic nonuniversal behavior can serve as a qualitative measure of the two-band character of ferromagnetic superconductors. The problem of the upper critical field determination is finally discussed.

\section{FERROMAGNETIC SUPERCONDUCTORS WITH TRIPLET PAIRING}

\section{A. Two-band superconductivity}

For a triplet superconductor the order parameter is written as [10]

$$
\Delta_{\alpha \beta}(\mathbf{R}, \mathbf{k})=\left(\begin{array}{cc}
\Delta_{\uparrow} & \Delta_{0} \\
\Delta_{0} & \Delta_{\downarrow}
\end{array}\right)=\left(\mathbf{d}^{\Gamma}(\mathbf{R}, \mathbf{k}) \boldsymbol{\sigma}\right) i \sigma_{y}
$$




$$
=\left(\begin{array}{cc}
-d_{x}(\mathbf{R}, \mathbf{k})+i d_{y}(\mathbf{R}, \mathbf{k}) & d_{z}(\mathbf{R}, \mathbf{k}) \\
d_{z}(\mathbf{R}, \mathbf{k}) & d_{x}(\mathbf{R}, \mathbf{k})+i d_{y}(\mathbf{R}, \mathbf{k})
\end{array}\right)
$$

where $\boldsymbol{\sigma}=\left(\sigma_{x}, \sigma_{y}, \sigma_{z}\right)$ are the Pauli matrices. The superconducting states $\mathbf{d}^{\Gamma}(\mathbf{R}, \mathbf{k})$ with different critical temperatures in the ferromagnetic crystals are classified in accordance with the irreducible co-representations $\Gamma$ of the magnetic group $M$ of the crystal [7-9]. All the co-representations in ferromagnets with orthorombic and cubic symmetries are one-dimensional. However, they obey multicomponent order parameters determined through the coordinate dependent pairing amplitudes: one per each band populated by electrons with spins "up" or "down" and one per each pair of the bands with the opposite spins (zero spin projection states). Owing to the big difference in the Fermi momenta, the pairing of electrons from the different bands is negligibly small. Hence, we shall neglect the pairing amplitude with zero spin projection; in another words $\Delta_{0}=d_{z}(\mathbf{R}, \mathbf{k})=0$ will be taken throughout the paper. Also, we limit ourself to the consideration of two band ferromagnetic superconductors with a strong spin-orbital coupling, when the two-component order parameter has the form

$$
\mathbf{d}^{\Gamma}(\mathbf{R}, \mathbf{k})=\frac{1}{2}\left[-(\hat{x}+i \hat{y}) \Delta_{\uparrow}(\mathbf{R}, \mathbf{k})+(\hat{x}-i \hat{y}) \Delta_{\downarrow}(\mathbf{R}, \mathbf{k})\right]
$$

which was first pointed out in the paper [11]. Here $\hat{x}, \hat{y}$ are the unit vectors of the spin (or, more exactly, of the pseudospin [10]) coordinate system pinned to the crystal axes.

$$
\Delta_{\uparrow}(\mathbf{R}, \mathbf{k})=-\eta_{1}(\mathbf{R}) f_{-}(\mathbf{k}), \quad \Delta_{\downarrow}(\mathbf{R}, \mathbf{k})=\eta_{2}(\mathbf{R}) f_{+}(\mathbf{k}) .
$$

The functions $f_{ \pm}(\mathbf{k})=f_{x}(\mathbf{k}) \pm i f_{y}(\mathbf{k})$ and the projections $f_{i}(\mathbf{k})$, with $i=x, y$ are odd functions of the momentum directions of the pairing particles on the Fermi surface. The general forms of these functions for the different corepresentations in ferromagnetic superconductors with orthorhombic and cubic symmetries are listed in the paper [7]. For instance, in the case of the $A_{1}$ representation in orthorombic crystal, they are

$$
f_{x}(\mathbf{k})=k_{x} u_{1}^{A_{1}}+i k_{y} u_{2}^{A_{1}}, \quad f_{y}(\mathbf{k})=k_{y} u_{3}^{A_{1}}+i k_{x} u_{4}^{A_{1}},
$$

where $u_{1}{ }^{A_{1}}, \ldots$ are real functions of $k_{x}{ }^{2}, k_{y}{ }^{2}, k_{z}{ }^{2}$. The simple consequence of this is that the nodes in the quasiparticle spectrum of the superconducting A-states in orthorombic ferromagnets dictated only by the symmetry are the nodes lying on the northern and southern poles of the Fermi surface $k_{x}=k_{y}=0$. On the contrary, for the B-states they are on the line of the equator $k_{z}=0$.

The order parameter amplitudes $\eta_{1}(\mathbf{R})$ and $\eta_{2}(\mathbf{R})$ (which are coordinate dependent and complex) have been considered (and discussed) in the paper [7] as being equal. This is in general not true. However, even in the general case, they are not completely independent:

$$
\eta_{1}(\mathbf{R})=\left|\eta_{1}(\mathbf{R})\right| e^{i \varphi(\mathbf{R})}, \quad \eta_{2}(\mathbf{R})=\left|\eta_{2}(\mathbf{R})\right| e^{i \varphi(\mathbf{R}) \pm i \pi} .
$$

Although being different by their modulous, they have the same phase with an accuracy $\pm \pi$. The latter property guarantees the consistency of the transformation of both parts of the order parameter under the time reversal.

The BCS Hamiltonian in a two-band ferromagnet with a triplet pairing is

$$
H=\sum_{\mathbf{k}, \mathbf{k}^{\prime}, \alpha}\left\langle\mathbf{k}\left|\hat{h}_{\alpha}\right| \mathbf{k}^{\prime}\right\rangle a_{\mathbf{k} \alpha}^{\dagger} a_{\mathbf{k}^{\prime} \alpha}+\frac{1}{2} \sum_{\mathbf{k}, \mathbf{k}^{\prime}, \mathbf{q}, \alpha, \beta} V_{\alpha \beta}\left(\mathbf{k}, \mathbf{k}^{\prime}\right) a_{-\mathbf{k}+\mathbf{q} / 2, \alpha}^{\dagger} a_{\mathbf{k}+\mathbf{q} / 2, \alpha}^{\dagger} a_{\mathbf{k}^{\prime}+\mathbf{q} / 2, \beta} a_{-\mathbf{k}^{\prime}+\mathbf{q} / 2, \beta},
$$

where the band indices $\alpha$ and $\beta$ are $(\uparrow, \downarrow)$ or $(1,2)$,

$$
\hat{h}_{\alpha}=\hat{\varepsilon}_{\alpha}-\mu_{B} \hat{\mathbf{g}}_{\alpha} \mathbf{H}_{\text {ext }} / 2+U(\mathbf{r})-\varepsilon_{F}
$$

are one particle band energy operators, the functions $\hat{\varepsilon}_{\alpha}$ (including the exchange splitting) and the $\hat{\mathbf{g}}_{\alpha}$-factor depend on the gauge invariant operator $-i \nabla+(e / c) \mathbf{A}(\mathbf{r})$ and on the crystallographic directions. In the simplest case of isotropic bands without a spin-orbital coupling, $\mathbf{g}_{1,2}= \pm 2 \mathbf{H}_{\text {ext }} / H_{\text {ext }} . U(\mathbf{r})$ is an impurity potential, $\mathbf{H}_{\text {ext }}$ is an external magnetic field,

$$
\nabla \times \mathbf{A}=\mathbf{B}=\mathbf{H}_{\mathrm{ext}}+4 \pi \mathbf{M},
$$

and $\mathbf{M}$ is the magnetic moment of the ferromagnet.

The pairing potential interaction is expanded as

$$
V_{\alpha \beta}\left(\mathbf{k}, \mathbf{k}^{\prime}\right)=-V_{\alpha \beta} \varphi_{\alpha}(\mathbf{k}) \varphi_{\beta}{ }^{*}\left(\mathbf{k}^{\prime}\right),
$$


where

$$
\varphi_{\uparrow}(\mathbf{k})=-f_{-}(\mathbf{k}), \quad \varphi_{\downarrow}(\mathbf{k})=f_{+}(\mathbf{k}) .
$$

It contains four different interaction terms which can be typically classified into two groups corresponding to: (i) a pairing between electrons with the same spin polarization (intraband interaction) and (ii) the interband scattering terms with $V_{\uparrow \downarrow}=V_{\downarrow \uparrow}$ describing the transitions of the pair electron from one sheet of the Fermi surface to the other sheet by reversing the pair spin orientation with the help of the spin-orbit coupling.

When the interband scattering is negligible $V_{\uparrow \downarrow}=V_{\downarrow \uparrow}=0$, the pairing of the electrons occurs first only in one of the sheets of the Fermi surface like in the $A_{1}$ phase of ${ }^{3} \mathrm{He}$. In general, the superconductivity in each band is not independent.

\section{B. Gor'kov equations}

We want to determine the Green's functions of ferromagnetic superconductors in the absence of external perturbations and impurity scattering. Even under these simple conditions, the system is not spatially uniform due to the inherent presence of $4 \pi M$. If we neglect $4 \pi M$, the system is spatially uniform. Then, we can write the Gor'kov equations in the form

$$
\begin{aligned}
& \left(i \omega_{n}-\xi_{\mathbf{k} \alpha}\right) G_{\alpha}\left(\mathbf{k}, \omega_{n}\right)+\Delta_{\alpha}(\mathbf{k}) F_{\alpha}^{\dagger}\left(\mathbf{k}, \omega_{n}\right)=1 \\
& \left(i \omega_{n}+\xi_{\mathbf{k} \alpha}\right) F_{\alpha}^{\dagger}\left(\mathbf{k}, \omega_{n}\right)+\Delta_{\alpha}^{\dagger}(\mathbf{k}) G_{\alpha}\left(\mathbf{k}, \omega_{n}\right)=0
\end{aligned}
$$

where $\xi_{\mathbf{k} \alpha}=\varepsilon_{\mathbf{k} \alpha}-\varepsilon_{F}$ and $\omega_{n}=\pi T(2 n+1)$ are Matsubara frequencies. The equations for each band are only coupled through the order parameter given by the self-consistency condition

$$
\Delta_{\alpha}(\mathbf{k})=-T \sum_{n} \sum_{\mathbf{k}^{\prime}} \sum_{\beta=\uparrow, \downarrow} V_{\alpha, \beta}\left(\mathbf{k}, \mathbf{k}^{\prime}\right) F_{\beta}\left(\mathbf{k}^{\prime}, \omega_{n}\right) .
$$

The superconductor Green's functions are

$$
\begin{aligned}
G_{\alpha}\left(\mathbf{k}, \omega_{n}\right) & =-\frac{i \omega_{n}+\xi_{\mathbf{k} \alpha}}{\omega_{n}^{2}+E_{\mathbf{k}, \alpha}^{2}} \\
F_{\alpha}\left(\mathbf{k}, \omega_{n}\right) & =\frac{\Delta_{\alpha}(\mathbf{k})}{\omega_{n}^{2}+E_{\mathbf{k}, \alpha}^{2}}
\end{aligned}
$$

where $E_{\mathbf{k}, \alpha}=\sqrt{\xi_{\mathbf{k} \alpha}^{2}+\left|\Delta_{\alpha}(\mathbf{k})\right|^{2}}$. Obviously, the superconductivity in ferromagnetic superconductors is non-unitary.

The Gor'kov equations taking into consideration the magnetic moment $4 \pi M$, an external field and non-magnetic point-like impurities can be easily written according to the general procedure described in [10]. We shall not overload the paper by this and just write the self-consistency equations near the superconducting transition.

\section{The order parameter equations near the superconducting transition}

This system consists of two equations for the order parameter components with spin polarizations "up" and "down",

$$
\begin{aligned}
\Delta_{\alpha}(\mathbf{R}, \mathbf{r})= & -T \sum_{n, \beta} \int d \mathbf{r}^{\prime} V_{\alpha, \beta}\left(\mathbf{r}, \mathbf{r}^{\prime}\right) G_{\beta}\left(\mathbf{r}^{\prime}, \tilde{\omega}_{n}^{\beta}\right) G_{\beta}\left(\mathbf{r}^{\prime},-\tilde{\omega}_{n}^{\beta}\right) \\
& \times \exp \left[i \mathbf{r}^{\prime} \mathbf{D}(\mathbf{R})\right]\left\{\Delta_{\beta}\left(\mathbf{R}, \mathbf{r}^{\prime}\right)+\Sigma_{\beta}\left(\tilde{\omega}_{n}^{\beta}, \mathbf{R}\right)\right\},
\end{aligned}
$$

and two equations for the impurity self-energy components

$$
\begin{aligned}
\Sigma_{\alpha}\left(\tilde{\omega}_{n}^{\alpha}, \mathbf{R}\right)= & n_{\mathrm{i}} u_{\alpha}^{2} \int d \mathbf{r} G_{\alpha}\left(\mathbf{r}, \tilde{\omega}_{n}{ }^{\alpha}\right) G_{\alpha}\left(\mathbf{r},-\tilde{\omega}_{n}{ }^{\alpha}\right) \exp [i \mathbf{r D}(\mathbf{R})] \\
& \times\left\{\Delta_{\alpha}(\mathbf{R}, \mathbf{r})+\Sigma_{\alpha}\left(\tilde{\omega}_{n}^{\alpha}, \mathbf{R}\right)\right\}
\end{aligned}
$$

where $\tilde{\omega}_{n}^{\alpha}=\omega_{n}+\operatorname{sign} \omega_{n} / 2 \tau_{\alpha}$, and $\tau_{\alpha}$ is the quasi-particle mean free time in the different bands. These mean free times are related in the Born approximation to the impurity concentration $n_{\mathrm{i}}$ through 


$$
\frac{1}{2 \tau_{\alpha}}=\pi n_{\mathrm{i}} N_{0 \alpha} u_{\alpha}^{2}
$$

with $u_{\alpha}$ the amplitude of the impurity scattering, and $N_{0 \alpha}$ the density of electronic states in each band.

The operator of covariant differentiation is

$$
\mathbf{D}(\mathbf{R})=-i \frac{\partial}{\partial \mathbf{R}}+\frac{2 e}{c} \mathbf{A}(\mathbf{R}) .
$$

The normal metal electron Green functions are

$$
G_{\alpha}\left(\mathbf{r}, \tilde{\omega}_{n}^{\alpha}\right)=\int \frac{d \mathbf{p}}{(2 \pi)^{3}} e^{i \mathbf{p} \cdot \mathbf{r}}\left(i \tilde{\omega}_{n}^{\alpha}-\xi_{\mathbf{p}, \alpha}+\mu_{B} \mathbf{g}_{\mathbf{p}, \alpha} \mathbf{H}_{\mathrm{ext}} / 2\right)^{-1}
$$

The order parameter components in different bands are determined in accordance with (3):

$$
\Delta_{\uparrow}(\mathbf{R}, \mathbf{r})=-\eta_{1}(\mathbf{R}) f_{-}(\mathbf{r}), \quad \Delta_{\downarrow}(\mathbf{R}, \mathbf{r})=\eta_{2}(\mathbf{R}) f_{+}(\mathbf{r}) .
$$

\section{The critical temperature $T_{c 0}$}

In the absence of an external field let us first find the critical temperature $T_{c 0}$ of a pure ferromagnetic superconductor in the (formally) spacially uniform situation of a negligible ferromagnetic moment $\mathbf{M}=0$. In this case the anomalous impurity self-energy part $\Sigma_{\alpha}\left(\widetilde{\omega}_{n}^{\alpha}, \mathbf{R}\right)=0$, and from (15) we obtain the system of equations

$$
\begin{aligned}
& \eta_{1}=\left(g_{1} \eta_{1}+g_{12} \eta_{2}\right) \lambda\left(T_{c 0}\right), \\
& \eta_{2}=\left(g_{21} \eta_{1}+g_{2} \eta_{2}\right) \lambda\left(T_{c 0}\right),
\end{aligned}
$$

where $g_{1}=V_{\uparrow \uparrow}\left\langle\left|f_{-}(\mathbf{k})\right|^{2} N_{0 \uparrow}(\hat{\mathbf{k}})\right\rangle$, the angular brackets mean the averaging over the Fermi surface, $N_{0 \uparrow}(\hat{\mathbf{k}})$ is the angular dependent density of electronic states at the Fermi surface of the band $\uparrow$. Correspondingly $g_{12}=V_{\uparrow \downarrow}\left\langle\left|f_{+}(\mathbf{k})\right|^{2} N_{0 \downarrow}(\hat{\mathbf{k}})\right\rangle$, $g_{21}=V_{\downarrow \uparrow}\left\langle\left|f_{-}(\mathbf{k})\right|^{2} N_{0 \uparrow}(\hat{\mathbf{k}})\right\rangle$, and $g_{2}=V_{\downarrow \downarrow}\left\langle\left|f_{+}(\mathbf{k})\right|^{2} N_{0 \downarrow}(\hat{\mathbf{k}})\right\rangle$. The function $\lambda(T)$ is

$$
\lambda(T)=2 \pi T \sum_{n \geq 0} \frac{1}{\omega_{n}}=\ln \frac{2 \gamma \epsilon}{\pi T},
$$

$\ln \gamma=0,577 \ldots$ is the Euler constant, $\epsilon$ is an energy cutoff.

Thus, similar to [1] the critical temperature is given by

$$
T_{c 0}=(2 \gamma \epsilon / \pi) \exp (-1 / g),
$$

where $g$ is defined by the maximum value for which the determinant of the system is zero (20)

$$
g=\left(g_{1}+g_{2}\right) / 2+\sqrt{\left(g_{1}-g_{2}\right)^{2} / 4+g_{12} g_{21}} .
$$

In particular, at $g_{12}, g_{21} \ll g_{1}, g_{2}$, the critical temperature is determined by

$$
g=\max \left(g_{1}, g_{2}\right) .
$$

\section{E. The critical temperature dependence on impurities concentration}

Triplet superconductivity is suppressed by non-magnetic impurities [12]. Moreover, the law of suppression of the superconductivity is described by the universal Abrikosov-Gor'kov (AG) dependence [13]

$$
-\ln t=\Psi\left(\frac{1}{2}+\frac{x}{4 \gamma t}\right)-\Psi\left(\frac{1}{2}\right)
$$

valid for any unconventional superconducting state and applicable in particular to a concrete unconventional superconductor independently of the pressure [10]. Here $\Psi$ is the digamma function. The variable $t=T_{c} / T_{c 0}$ is the ratio of 
the critical temperature of the superconductor with a given concentration of impurities $n_{\mathrm{i}}$ to the critical temperature of the clean superconductor, and $x=n_{\mathrm{i}} / n_{\mathrm{ic}}=\tau_{\mathrm{c}} / \tau$ is the ratio of the impurity concentration in the superconductor to the critical impurity concentration destroying superconductivity, or the inverse ratio of the corresponding mean free particle lifetimes. The critical mean free time is given by $\tau_{c}=\gamma / \pi T_{c 0}$. This dependence has been demonstrated (although with some dispersion of the experimental points) for the triplet superconductor $\mathrm{Sr}_{2} \mathrm{RuO}_{4}$ [14].

Deviations from the universality of the AG law can be caused by the anisotropy of the scattering which takes place in the presence of extended imperfections in the crystal. Such a modification of the theory applied to $\mathrm{UPt}_{3}$ has been considered previously [15]. However, a complete experimental investigation of the suppression of superconductivity by impurities in this unconventional superconductor, in particular the study of the universality of the behavior, has not been performed.

The nonuniversality of the suppression of superconductivity can also be caused by any inelastic scattering mechanism by impurities with internal degrees of freedom of magnetic or nonmagnetic origin. For the simplest discussion of this, see $[16]$.

Finally, universality is certainly not expected in multiband superconductors. Theories for this case have been developed with regard to the unconventional superconductivity in $\mathrm{Sr}_{2} \mathrm{RuO}_{4}$ (p-wave, two-band two-dimensional model [17]) and conventional superconductivity in $\mathrm{MgB}_{2}$ (anisotropic scattering two-band model [18]).

A simple modification of the universal AG law for the suppression of the superconductivity by impurities in a two-band ferromagnetic superconductor is derived here. Our consideration is limited to the simplest case of scattering by ordinary point-like impurities. Then, due to spin conservation, one can neglect interband quasi-particle scattering and take into account only the intraband quasi-particle scattering on impurities. At finite impurity concentration the system of equations similar to (20) is:

$$
\begin{aligned}
& \eta_{1}=g_{1} \Lambda_{1}(T) \eta_{1}+g_{12} \Lambda_{2}(T) \eta_{2}, \\
& \eta_{2}=g_{21} \Lambda_{1}(T) \eta_{1}+g_{2} \Lambda_{2}(T) \eta_{2},
\end{aligned}
$$

where

$$
\Lambda_{1,2}(T)=\Psi\left(\frac{1}{2}\right)-\Psi\left(\frac{1}{2}+\frac{1}{4 \pi \tau_{1,2} T}\right)+\ln \frac{T_{c 0}}{T}+\lambda\left(T_{c 0}\right) .
$$

Hence, the critical temperature is determined from the equation

$$
\left(g_{1} \Lambda_{1}(T)-1\right)\left(g_{2} \Lambda_{2}(T)-1\right)-g_{12} g_{21} \Lambda_{1}(T) \Lambda_{2}(T)=0 .
$$

In particular, at $g_{12}, g_{21} \ll g_{1}, g_{2}$ the critical temperature is determined by the $\max \left(T_{c 1}, T_{c 2}\right)$ of the solutions of the equations

$$
\begin{aligned}
& \ln \frac{T_{c 0}}{T_{c 1}}=\Psi\left(\frac{1}{2}+\frac{1}{4 \pi \tau_{1} T_{c 1}}\right)-\Psi\left(\frac{1}{2}\right)+\frac{1}{g_{1}}-\lambda\left(T_{c 0}\right), \\
& \ln \frac{T_{c 0}}{T_{c 2}}=\Psi\left(\frac{1}{2}+\frac{1}{4 \pi \tau_{2} T_{c 2}}\right)-\Psi\left(\frac{1}{2}\right)+\frac{1}{g_{2}}-\lambda\left(T_{c 0}\right) .
\end{aligned}
$$

Let us accept for determination that $g_{1}>g_{2}$. Hence, the maximal critical temperature in the absence of impurities is defined by $1 / g_{1}=\lambda\left(T_{c 0}\right)$. Then, at small impurity concentrations the solutions of (29) and (30) are the linear functions of impurities concentration:

$$
\begin{gathered}
T_{c 1}=T_{c 0}-\frac{\pi}{8 \tau_{1}}, \\
T_{c 2}=T_{c 0}-\frac{1}{g_{2}}+\frac{1}{g_{1}}-\frac{\pi}{8 \tau_{2}} .
\end{gathered}
$$

These lines can in principle intersect each other. As a result, an upturn in the critical temperature dependence on impurity concentration $T_{c}\left(n_{i}\right)$ appears. Such a type of deviation of the $T_{c}\left(n_{i}\right)$ dependence from the AG-law presents the direct manifestation of the two-band character of the superconductivity. On the other hand, an absence of strong deviations from the universal one-band curve if found experimentally in a ferromagnetic superconductor would mean that the superconductivity is developed in one-band with only electrons with "up" spins paired and the "down" spin electrons leave normal (or vice versa). 
Another specific feature of the ferromagnetic superconductors is that even in the absence of an external magnetic field the exchange field $H_{\mathrm{ex}} \sim E_{\mathrm{ex}} / \mu_{B}$ acting on the electron spins in a ferromagnet produces in addition an electromagnetic field $4 \pi M \sim 4 \pi \mu_{B} k_{F}{ }^{3}$ acting via the electronic charges on the orbital motion of electrons, and suppressing the superconductivity [19]. Hence, the actual critical temperature in ferromagnetic superconductors is always smaller by the value $\sim 4 \pi M / H_{c 2}(T=0)$ relative to the (imaginary) ferromagnetic superconductor without $4 \pi M$. The upper critical field $H_{c 2}$ is also purity dependent. That is why the impurity concentration dependence of the actual $T_{c}$ in a ferromagnetic superconductor might be determined not only directly by the suppression of superconducting correlations by the impurity scattering as in any nonconventional superconductor but also indirectly through the supression of $H_{c 2}$. In fact the second indirect mechanism has a negligible influence because the ratio $4 \pi M / H_{c 2}(T=0)$ is of the order of $10^{-2}$ for superconductors with an upper critical field of the order of several Teslas.

Thus, the problem of the determination of the critical temperature in a superconducting ferromagnet is at bottom the problem of the determination of the upper critical field in a single domain ferromagnet.

\section{F. The upper critical field}

The equations for the determination of the upper critical field at least near $T_{c}$ are easily derived from the system (15)-(16). Keeping only the lowest order gradient terms we have

$$
\begin{aligned}
\Delta_{\alpha}(\mathbf{R}, \mathbf{r})= & -T \sum_{n, \beta} \int d \mathbf{r}^{\prime} V_{\alpha, \beta}\left(\mathbf{r}, \mathbf{r}^{\prime}\right) G_{\beta}\left(\mathbf{r}^{\prime}, \tilde{\omega}_{n}^{\beta}\right) G_{\beta}\left(\mathbf{r}^{\prime},-\tilde{\omega}_{n}^{\beta}\right) \\
& \times\left\{\left(1-\left(\mathbf{r}^{\prime} \mathbf{D}(\mathbf{R})\right)^{2} / 2\right) \Delta_{\beta}\left(\mathbf{R}, \mathbf{r}^{\prime}\right)+\left(i \mathbf{r}^{\prime} \mathbf{D}(\mathbf{R})\right) \Sigma_{\beta}\left(\tilde{\omega}_{n}^{\beta}, \mathbf{R}\right)\right\},
\end{aligned}
$$

and

$$
\begin{aligned}
\Sigma_{\alpha}\left(\tilde{\omega}_{n}^{\alpha}, \mathbf{R}\right)= & n_{\mathrm{i}} u_{\alpha}^{2} \int d \mathbf{r} G_{\alpha}\left(\mathbf{r}, \tilde{\omega}_{n}^{\alpha}\right) G_{\alpha}\left(\mathbf{r},-\tilde{\omega}_{n}^{\alpha}\right) \\
& \times\left\{(i \mathbf{r D}(\mathbf{R})) \Delta_{\alpha}(\mathbf{R}, \mathbf{r})+\Sigma_{\alpha}\left(\tilde{\omega}_{n}^{\alpha}, \mathbf{R}\right)\right\} .
\end{aligned}
$$

Finding $\Sigma_{\alpha}\left(\tilde{\omega}_{n}^{\alpha}, \mathbf{R}\right)$ from the last equation and substituting it into (33), we obtain after all the necessary integrations the pair of the Ginzburg-Landau equations for the two components of the order parameter

$$
\begin{aligned}
& \eta_{1}=V_{\uparrow \uparrow} \hat{\alpha}_{1} \eta_{1}+V_{\uparrow \downarrow} \hat{\alpha}_{2} \eta_{2}, \\
& \eta_{2}=V_{\uparrow \downarrow} \hat{\alpha}_{1} \eta_{1}+V_{\downarrow \downarrow} \hat{\alpha}_{2} \eta_{2},
\end{aligned}
$$

where the operator $\hat{\alpha}_{1}$ consists of the previously determined homogeneous part and of the second order gradient terms

$$
\hat{\alpha}_{1}=\left\langle\left|f_{-}(\mathbf{k})\right|^{2} N_{0 \uparrow}(\hat{\mathbf{k}})\right\rangle \Lambda_{1}(T)-K_{\uparrow i j} D_{i} D_{j}
$$

The gradient terms coefficients are

$$
\begin{aligned}
K_{\uparrow i j} & =\left\langle\left|f_{-}(\mathbf{k})\right|^{2} N_{0 \uparrow}(\hat{\mathbf{k}}) v_{F \uparrow i}(\hat{\mathbf{k}}) v_{F \uparrow j}(\hat{\mathbf{k}})\right\rangle \frac{\pi T}{2} \sum_{n \geq 0} \frac{1}{\left|\tilde{\omega}^{\uparrow} n\right|^{3}} \\
& +\left\langle f_{-}(\mathbf{k}) N_{0 \uparrow}(\hat{\mathbf{k}}) v_{F \uparrow i}(\hat{\mathbf{k}})\right\rangle\left\langle f_{-}^{*}(\mathbf{k}) N_{0 \uparrow}(\hat{\mathbf{k}}) v_{F \uparrow j}(\hat{\mathbf{k}})\right\rangle \frac{\pi^{2} T n_{i} u_{\uparrow}{ }^{2}}{2} \sum_{n \geq 0} \frac{1}{\omega_{n}^{2} \tilde{\omega}^{\uparrow} n^{2}}
\end{aligned}
$$

The operator $\hat{\alpha}_{2}$ is obtained from (36)-(37) by the natural substitutions $1 \rightarrow 2, \uparrow \rightarrow \downarrow,+\rightarrow-$.

Now, the problem of the upper critical field finding is just the resolution of the two coupled equations (35). There are a lot of different situations depending on the crystal symmetry, the direction of spontaneous magnetization and on the external field orientation. The simplest case is when the external magnetic field is parallel or antiparallel to the easy magnetization axis. If this latter coincides with the 4-th order symmetry axis in the cubic crystal as it is the case in $\mathrm{ZrZn}_{2}$, then the gradient terms in the perpendicular plane are isotropic and described by the two constants $K_{\uparrow i j}=K_{\uparrow} \delta_{i j}$ and $K_{\downarrow i j}=K_{\downarrow} \delta_{i j}$. This case formally corresponds to the problem of the determination of the upper critical field parallel to the c-direction in the two-band hexagonal superconductor $\mathrm{MgB}_{2}$ solved in [20]. Then, the linearized Ginzburg-Landau equations describe a system of two coupled oscillators and have their solution in the form $\eta_{1}=c_{1} f_{0}(x)$ and $\eta_{2}=c_{2} f_{0}(x)$, where $f_{0}(x)=\exp \left(-h x^{2} / 2\right)$, and $h$ is related to the upper critical field by means of 


$$
\left|H_{c 2} \pm 4 \pi M\right|=\frac{h(\tau) \Phi_{0}}{2 \pi},
$$

where $\Phi_{0}$ is the flux quantum.

Let us for simplicity limit ourself to the impurityless case. Then, $\tau=1-T / T_{c 0}$ and the equation for the determination of the upper critical field is

$$
\begin{gathered}
{\left[g_{1}\left(\tau+\lambda\left(T_{c 0}\right)\right)+V_{\uparrow \uparrow} K_{\uparrow} h-1\right]\left[g_{2}\left(\tau+\lambda\left(T_{c 0}\right)\right)+V_{\downarrow \downarrow} K_{\downarrow} h-1\right]} \\
-\left[g_{12}\left(\tau+\lambda\left(T_{c 0}\right)\right)+V_{\uparrow \downarrow} K_{\uparrow} h-1\right]\left[g_{21}\left(\tau+\lambda\left(T_{c 0}\right)\right)+V_{\uparrow \downarrow} K_{\downarrow} h-1\right]=0 .
\end{gathered}
$$

This is a simple square equation and, as before, if we consider the case $g_{12}, g_{21} \ll g_{1}, g_{2}$ and $g_{1}>g_{2}$, then we obtain the two following roots

$$
\begin{gathered}
h_{1}(\tau)=\frac{g_{1} \tau}{V_{\uparrow \uparrow} K_{\uparrow}}, \\
h_{2}(\tau)=\frac{g_{2}}{V_{\downarrow \downarrow} K_{\downarrow}}\left(\tau+\frac{1}{g_{1}}-\frac{1}{g_{2}}\right) .
\end{gathered}
$$

This two lines can in principle intersect each other. As a result, an upturn in the temperature dependence of the upper critical field appears.

In the most anisotropic situation such as in the orthorombic crystals $\mathrm{UGe}_{2}$ and URhGe, all the coefficients $K_{\uparrow x x}$, $K_{\uparrow y y}, K_{\downarrow x x}$ and $K_{\downarrow y y}$ are different, even for an external field direction parallel or antiparallel to the easy magnetization axis. Then, our system of equations can be solved following a variational approach developed in [20]. Again, an upturn in the $h(\tau)$ dependence can be possible.

The comparison with an experiment masked by the presence of many ferromagnetic domains will be not easy. Monodomain measurements are possible in high enough fields. To work in this region one can easily obtain the fourth order gradient terms contributing to the Ginzburg-Landau equations. However, the problem of the theoretical determination of the upper critical field at arbitrary temperature has the same principal difficulty as in any conventional anisotropic superconductor [21].

\section{CONCLUSION}

Ferromagnetic superconductors are in general multiband metals. The two-band description of ferromagnetic superconductors with a triplet pairing developed in this paper presents the simplest model applicable to this type of material. One-band superconductivity in these superconductors arises only at a negligibly small spin-orbit coupling, as it is the case for the $A_{1}$-phase of ${ }^{3} \mathrm{He}$. We studied the dependence of the critical temperature $T_{c}$ on the concentration of ordinary point-like impurities in the framework of a two-band weak coupling BCS theory. We demonstrated that the non-universal $T_{c}(x)$ dependence could serve as a qualitative measure of the two band character of the superconductivity in ferromagnetic superconductors. Also, the general equations for the determination of the upper critical field at arbitrary temperature and impurity concentration were derived. The solution of these equations near the critical temperature was found in the simplest case of a cubic crystalline symmetry for the field orientation parallel or antiparallel to the 4 -th order symmetry axis.

\section{ACKNOWLEDGEMENTS}

The authors are indebted to Dr. M. Zhitomirskii for the valuable discussions about the physics of multi-band superconductivity.

[1] H. Suhl, B.T. Matthias, and L.R. Walker, Phys. Rev. Lett. 3, 552 (1959).

[2] See the special issue of Physica C 385, 1-305 (2003). 
[3] S.S. Saxena, P. Agarval, K. Ahilan, F.M. Grosche, R.K.W. Hasselwimmer, M.J. Steiner, E. Pugh, I.R. Walker, S.R. Julian, P. Monthoux, G.G. Lonzarich, A. Huxley, I. Sheikin, D. Braithwaite, and J. Flouquet, Nature 406, 587 (2000).

[4] A. Huxley, I. Sheikin, E. Ressouche, N. Kernavanois, D. Braithwaite, R. Calemczuk, and J. Flouquet, Phys. Rev. B 63, 144519 (2001).

[5] C. Pfleiderer, M. Uhlarz, S. Heiden, R. Vollmer, H.v. Lohneysen, N.R. Bernhoeft, and G.G. Lonzarich, Nature 412, 58 (2001).

[6] D. Aoki, A. Huxley, E. Ressouche, D. Braithwaite, J. Flouquet, J.-P. Brison, E. Lhotel and C. Paulsen, Nature 413, 613 (2001).

[7] V.P. Mineev, Phys. Rev. B 66, 134504 (2002).

[8] K.V. Samokhin and M.B. Walker, Phys. Rev. B 66, 024512 (2002).

[9] I.A. Fomin, Zh. Eksp. Teor. Fiz. 122, 1089 (2002) [Sov. Phys. JETP 95, 940 (2002)].

[10] V.P. Mineev and K.V. Samokhin, Introduction to Unconventional Superconductivity (Gordon and Breach, New York, 1999).

[11] K.V. Samokhin and M.B. Walker, Phys. Rev. B 66, 174501 (2002).

[12] A.I. Larkin, Pis'ma ZhETF 2, 205 (1965) [JETP Letters 2, 130 (1965)].

[13] A.A. Abrikosov, L.P. Gor'kov, ZhETF 39, 1781 (1960) [Sov. Phys. JETP 12, 1243 (1961)].

[14] A.P. Mackenzie, R.K.W. Haselwimmer, A.W. Tyler, G.G. Lonzarich, Y. Mori, S. Nishizaki, and Y. Maeno, Phys. Rev. Lett. 80, 161 (1998).

[15] J.B. Kycia, J.I. Hong, M.J. Graf, J.A. Sauls, D.N. Seidman, and W.P. Halperin, Phys.Rev. B 58, 603 (1998).

[16] V.P. Mineev, Pis'ma ZhETF 51, 399 (1990) [JETP Letters 51, 453 (1990)].

[17] D.F. Agterberg, Phys. Rev. B 60, R749 (1999).

[18] I.I. Mazin, O.K. Andersen, O. Jepsen, O.V. Dolgov, J. Kortus, A.A. Golubov, A.B. Kuz'menko, and D. van der Marel, Phys. Rev. Lett. 89, 107002 (2002).

[19] This is correct of course only if $4 \pi M>H_{c 1}$ (where $H_{c 1}$ is the lower critical field).

[20] M.E. Zhitomirskii and V.-H. Dao, cond-mat/0309372 (2003).

[21] P.C. Hohenberg and N.R. Werthamer, Phys. Rev. 153, 493 (1967). 